\title{
Diagnosis and Management of Barrett's Esophagus: A Retrospective Study Comparing the Endoscopic Assessment of Early Esophageal Lesions in the Community versus a Specialized Center
}

\author{
Erin Rayner-Hartley, Oliver Takach, Cherry Galorport, and Robert A. Enns \\ Department of Medicine, Division of Gastroenterology, University of British Columbia, Vancouver, Canada \\ Correspondence should be addressed to Robert A. Enns; rob.enns@ubc.ca
}

Received 29 May 2015; Accepted 26 July 2015

Copyright (C) 2016 Erin Rayner-Hartley et al. This is an open access article distributed under the Creative Commons Attribution License, which permits unrestricted use, distribution, and reproduction in any medium, provided the original work is properly cited.

\begin{abstract}
Specialized endoscopic evaluation for patients with Barrett's esophagus (BE) is well supported; however, no studies have shown that centers with expertise provide better quality care for BE with high-grade dysplasia or early adenocarcinoma. In this study, the investigators aimed to evaluate the management and clinical course for patients treated in a community practice versus a specialized $\mathrm{BE}$ center. Methods. A retrospective analysis of referrals from the community to our specialized center for evaluation of $\mathrm{BE}$ at $\mathrm{St}$ Paul's Hospital Division of Gastroenterology between January 2007 and February 2014 was performed. Subjects were patients who were referred for $\mathrm{BE}$ and dysplasia and subsequently reevaluated by endoscopy. The pathology and endoscopy reports from the community and our center were reviewed. Inclusion criteria were as follows: being $\geq 19$ years old and pathologic diagnosis of BE or dysplasia in the community. Exclusion criteria were as follows: incomplete pathology data or incomplete endoscopy reports from the community physicians. Results. A total of 77 patients were reviewed. The staging of $28.9 \%$ of patients referred from the community was changed from the initial pathological diagnosis. $18.4 \%$ of these patients were upstaged. Using Fischer's exact test, we showed that, in our specialized center, endoscopic impressions correlated significantly with pathology results $(p<0.0001)$.
\end{abstract}

\section{Introduction}

Barrett's esophagus (BE), a metaplastic transformation of the distal esophagus [1], affects $2 \%$ of the population in developed countries [2] and this incidence is rising [3]. Risk factors for $\mathrm{BE}$ include gastroesophageal reflux disease (GERD), age of 50 years or older, male sex, race, hiatal hernia, and elevated BMI [4-6].

In $\mathrm{BE}$, the progression from metaplasia to dysplasia and finally adenocarcinoma (AC) has been well established. Dysplasia is subclassified as indeterminate dysplasia, lowgrade dysplasia (LGD), or high-grade dysplasia (HGD) [4]. HGD, involving significant distortion of the glandular crypts, is at risk of progression to $\mathrm{AC}$. The classification of $\mathrm{AC}$ into intramucosal carcinoma (IMC) versus submucosal carcinoma (SMC) is necessary in order to choose the appropriate management plan (typically endoscopic versus surgical management).
The prevalence of $\mathrm{AC}$ in patients with $\mathrm{BE}$ and HGD was previously reported to be as high as $40 \%[7,8]$. More recent studies have shown significantly lower rates of invasive AC, $12.7 \%$ and $11.7 \%$, respectively, in patients who underwent esophagectomy for BE and HGD $[4,9]$. This overestimate was attributed to the lack of strict pathological definitions of invasive disease. IMC carries a low risk (3-4\%) of nodal involvement in contrast to SMC, which carries an $8-33 \%$ risk [10].

The management of patients with HGD and AC in $\mathrm{BE}$ has evolved in recent years. Surgical esophagectomy was previously the standard of care [8]. The risk of mortality in esophagectomy ranges from 3 to $8 \%$ [4]. Shaheen et al. introduced radiofrequency ablation (RFA) as an effective technique for eradicating dysplastic BE in 2009 [11]. Numerous studies have demonstrated better outcomes with endoscopic management of HGD and IMC in BE $[4,12-14]$. An endoscopic approach is now recognized as 
the standard of care for HGD and AC confined to the mucosa $[15,16]$.

Differentiating IMC from SMC is imperative in deciding whether endoscopic therapy is appropriate for patients with $\mathrm{AC}$ in BE. Once visible mucosal abnormalities (VMAs) have been identified, removal of these lesions using endoscopic mucosal resection (EMR) is pivotal for assessment and staging. Correctly identifying and removing IMC while excluding SMC is imperative prior to starting radiofrequency ablation (RFA) [17]. RFA does not provide tissue specimens and thus if submucosal invasion is present, other therapies should be considered. Endoscopic imaging techniques and adherence to biopsy protocols, such as Seattle protocol, have been shown to improve the identification of VMAs that contain HGD or early AC [16]. The use of high definition white light endoscopy (HD-WLE) and narrow band imaging (NBI) increases pathology detection $[18,19]$. A recent prospective randomized controlled trial comparing HD-WLE using Seattle protocol with NBI targeted biopsies showed that NBI has similar metaplasia detection rates while requiring fewer biopsies [20].

Specialized endoscopic evaluation for patients with BE is thus well supported, and practice norms of gastroenterologists have been studied. Singh et al. found that only one-third of gastroenterologists use HD-WLE and NBI when assessing $\mathrm{BE}$ [21]. Adherence to Seattle protocol for biopsies has been reported between 30 and $50 \%$ in the community [22, 23]. These findings suggest that the detection of VMA and early AC may vary depending on the clinical setting. At this time, there are limited studies that have shown that centers with expertise provide different assessments for BE patients with HGD or early AC [24].

A recent Australian prospective cohort study compared detection rates of mucosal lesions and early AC in dysplastic $\mathrm{BE}$ patients seen in the community versus a specialized $\mathrm{BE}$ unit [25]. 69 patients were referred and reassessed with HDWLE, NBI, Seattle protocol biopsies, and EMR when appropriate. They found a $56 \%$ increased cancer detection rate and suggested that patients with dysplastic BE be considered for referral to a specialized unit.

There is a risk that mucosal lesions harboring dysplasia and cancer may be missed on surveillance endoscopy. We retrospectively compared the endoscopic impression and pathology report in patients with esophageal abnormalities (ranging from intestinal metaplasia and dysplasia to carcinoma) assessed in the community and our specialized $\mathrm{BE}$ center. We aimed to compare the overall change in endoscopic impression, which could influence the physician's biopsy plan on initial view. We also aimed to compare the overall change in pathology leading to final diagnosis and patient management.

\section{Methods}

2.1. Referrals. We performed a retrospective analysis of referrals from the community to our center for evaluation between 01/07 and 02/14. Physicians specializing in gastroenterology, general surgery, and internal medicine made referrals to our
TABLE 1: Referral details.

\begin{tabular}{lc}
\hline & Number \\
\hline Median age, y & 64.5 \\
Gender & \\
M & 8 \\
F & 54 \\
Reason for referral & \\
BE & 19 \\
Dysplasia & 37 \\
Carcinoma & 5 \\
Other & 1 \\
Referring physician & \\
Gastroenterologist & 14 \\
Surgeon & 37 \\
General internist & 11 \\
\hline
\end{tabular}

center. Subjects included patients referred for BE, dysplasia, and $\mathrm{AC}$ who were subsequently reevaluated by endoscopy. The majority of these patients were referred from outside downtown Vancouver area. The pathology and endoscopy reports from the community and our center were reviewed.

Inclusion criteria included being $\geq 19$ years old and minimum pathologic diagnosis of BE in the community. Exclusion criteria included incomplete pathology data or endoscopy reports from referring physicians.

2.2. Assessment. One experienced endoscopist (RE) performed all examinations on the cohort of subjects. All exams were performed using an Olympus Gastroscope (GIF1TQ160 and/or GIF H180). Biopsies were taken according to Seattle protocol and described using the Prague classification.

2.3. Endoscopy Details. Endoscopic impression was retrospectively collected from the initial reports of referring gastroenterologists, surgeons, or internal medicine physicians. This was compared with the endoscopic impression at time of initial evaluation by our specialized gastroenterologist (RE).

2.4. Histology Details. One physician (ERH) retrospectively reviewed all referral histopathology reports. More than one specialized pathologist at our institution reviewed all histological findings, unless there was clear evidence of carcinoma on initial evaluation.

\section{Results}

77 patients were referred during this 7 -year retrospective review. 62 patients ( 54 males and 8 females) had sufficient data to be included in the study. Demographic and referral information is summarized in Table 1.

Endoscopic impression was categorized as follows: non$\mathrm{BE}, \mathrm{BE}$, dysplasia, and carcinoma. After assessment in our center, these impressions were changed $56 \%$ of the time (35/62 subjects). Overall, $52 \%$ of subjects (32/62) were upgraded from the referral impression, 5\% (3/62) were 
TABLE 2: Comparison of endoscopic impression at referral and after specialized center evaluation.

\begin{tabular}{|c|c|c|c|c|}
\hline & Non-BE & $\mathrm{BE}$ & Dysplasia & Carcinoma \\
\hline \multicolumn{5}{|l|}{ Endoscopic impression } \\
\hline At referral & 9 & 49 & 3 & 1 \\
\hline After specialized center evaluation & 1 & 33 & 21 & 7 \\
\hline \multicolumn{5}{|l|}{ Outcomes of referral endoscopic impression } \\
\hline Concordant & 0 & 25 & 1 & 1 \\
\hline Downgraded & 0 & 1 & 2 & 0 \\
\hline Upgraded & 9 & 23 & 0 & 0 \\
\hline
\end{tabular}

TABLE 3: Comparison of most advanced pathology at referral and after specialized center evaluation.

\begin{tabular}{|c|c|c|c|c|c|c|}
\hline & IM & LGD & HGD & IMC & SMC & Normal \\
\hline \multicolumn{7}{|l|}{ Pathology } \\
\hline At referral & 16 & 13 & 28 & 4 & 1 & 0 \\
\hline After specialized center evaluation & 18 & 7 & 29 & 4 & 3 & 1 \\
\hline \multicolumn{7}{|l|}{ Outcomes of referral pathology } \\
\hline Concordant & 14 & 4 & 21 & 2 & 1 & 0 \\
\hline Downgraded & 1 & 2 & 3 & 2 & 0 & 0 \\
\hline Upgraded & 1 & 7 & 4 & 0 & 0 & 0 \\
\hline
\end{tabular}

downgraded, and 43\% (27/62) remained the same. Results are summarized in Table 2.

Referral pathology was categorized as follows: intestinal metaplasia (IM), LGD, HGD, IMC, SMC, and normal. After endoscopic assessment, biopsy, and pathology review in our center, final diagnosis was changed $32 \%$ of the time (20/62). $19 \%(12 / 62)$ of subjects were upgraded, $13 \%(8 / 62)$ were downgraded, and 68\% (42/62) remained the same as referral. Results are summarized in Table 3.

Subjects with a different pathologic diagnosis after evaluation $(32 \%(20 / 62))$ were assessed. The majority of these subjects $(45 \%(9 / 20))$ were changed from the LGD group. Otherwise, 35\% (7/20) were changed from HGD and $10 \%$ $(2 / 20)$ changed from IM and IMC, respectively.

Lastly, outcomes of all patients were assessed. Interestingly, of the $19 \%$ (12/62) of upgraded subjects, $58 \%$ (7/12) were upstaged to HGD. The majority of these subjects $(86 \%(6 / 7))$ were treated endoscopically with EMR and/or RFA. The outcomes of patients with upgraded pathology are outlined in Table 4.

The correlation between the specialized physician's impression (RE) and pathology at our center was evaluated. Fischer's exact test was used to compare categories of $\mathrm{BE}$ and LGD to HGD and AC. We showed that our endoscopic impressions correlated significantly with pathology results $(p<0.0001)$.

\section{Discussion}

This is a single-center retrospective review of patients referred from the community to an advanced endoscopy center for BE, dysplasia, or AC. Esophageal mucosal abnormalities are often subtle and can be challenging to identify. The systematic assessment of the BE segment, including visualization with
HD-WLE, NBI, and sampling according to Seattle protocol, increases detection rate [22].

There are several important findings of this study. Firstly, endoscopic impression changed significantly when assessed by an endoscopist experienced in dysplasia. There are several potential explanations for this: imaging equipment (although many communities now have more advanced equipment than tertiary care centers), experience ("one sees only what one is looking for"), or spending more time to carefully analyze the esophagus.

Secondly, pathology and thus final diagnosis were changed in one-third of cases. Interestingly, LGD was the pathological diagnosis which changed most often, representing $45 \%$ of changed cases. Possible reasons for this significant number include the following: adherence to biopsy protocols and techniques allowing for improved samples (including the ability to perform EMR at our center), experienced visualization of lesions leading to appropriate sampling, or the specialized assessment by a GI pathologist.

Lastly, our outcomes are compatible with the current standard of care for BE, dysplasia, and IMC, which includes endoscopic assessment and/or treatment. Among subjects with an upgrade in pathological diagnosis, endoscopic treatment was possible in the vast majority of cases $(92 \%, 11 / 12)$. This represents the most important group of subjects assessed in this cohort, those at high risk of progression to carcinoma who may have been missed on initial evaluation.

There is minimal data showing that centers with experience in BE provide better care for BE patients with $\mathrm{HGD} / \mathrm{CA}$ [25]. This study reports a significant benefit in detecting and managing patients with dysplasia. Endoscopists in specialized centers receiving referrals for BE have the advantage of a focused area of expertise, an initial assessment completed by colleagues in the community, a high degree of suspicion, 
TABLE 4: Outcomes and management of patients with upgraded pathology.

\begin{tabular}{lcr}
\hline Upgraded pathology & Outcome & Management \\
\hline IM, $n=1$ & 1 upgraded to LGD & 1, endoscopic therapy (EMR and/or RFA) \\
\hline LGD, $n=7$ & 7 upgraded to HGD & $\begin{array}{r}\text { 1, endoscopic surveillance and conservative management (ex PPI) } \\
\text { 6, endoscopic therapy (EMR and/or RFA) }\end{array}$ \\
\hline HGD, $n=4$ & $\begin{array}{l}2 \text { upgraded to IMC } \\
2 \text { upgraded to SMC }\end{array}$ & 4, endoscopic therapy (EMR and/or RFA) \\
\hline
\end{tabular}

and the luxury of additional time dedicated for evaluation of lesions.

The current expert consensus suggests that patients with $\mathrm{BE}$ and at least HGD be managed by gastroenterologists in a center where significant experience and exposure to this disorder exist [24]. Cameron et al. recently showed that patients with at least dysplastic BE were more appropriately investigated in a specialized BE unit. The current study supports this finding.

\section{Conclusion}

Referral and assessment of patients with BE at a specialized center significantly changed initial diagnosis and thus management. This study supports consideration of referral for specialized endoscopic assessment and pathology review. As identified in this review, a small but significant number of patients will likely benefit from early intervention with curative intent.

\section{Ethical Approval}

An application to the ethics board was approved for this retrospective chart review.

\section{Competing Interests}

The authors declare that they have no competing interests.

\section{Authors' Contributions}

Oliver Takach and Cherry Galorport should be titled research assistants that assisted in study design, statistics, presentation, and preparation of the paper.

\section{References}

[1] N. Vakil, S. V. van Zanten, P. Kahrilas et al., "The Montreal definition and classification of gastroesophageal reflux disease: a global evidence-based consensus," The American Journal of Gastroenterology, vol. 101, no. 8, pp. 1900-1920, 2006.

[2] J. B. Taylor and J. H. Rubenstein, "Meta-analyses of the effect of symptoms of gastroesophageal reflux on the risk of Barrett's esophagus," The American Journal of Gastroenterology, vol. 105, no. 8, pp. 1730-1737, 2010.

[3] P. Moayyedi and A. T. R. Axon, "Review article: Gastrooesophageal reflux disease - the extent of the problem," Alimentary Pharmacology and Therapeutics, Supplement, vol. 22, no. 1, pp. 11-19, 2005.
[4] J. Y. Nasr and R. E. Schoen, "Prevalence of adenocarcinoma at esophagectomy for Barrett's esophagus with high grade dysplasia," Journal of Gastrointestinal Oncology, vol. 2, no. 1, pp. 34-38, 2011.

[5] American Gastroenterological Association, S. J. Spechler, P. Sharma, R. F. Souza, J. M. Inadomi, and N. J. Shaheen, "American Gastroenterological Association medical position statement on the management of Barrett's esophagus," Gastroenterology, vol. 140, no. 3, pp. 1084-1091, 2011.

[6] G. W. Falk, T. W. Rice, J. R. Goldblum, and J. E. Richter, "Jumbo biopsy forceps protocol still misses unsuspected cancer in Barrett's esophagus with high-grade dysplasia," Gastrointestinal Endoscopy, vol. 49, no. 2, pp. 170-176, 1999.

[7] M. J. Edwards, D. R. Gable, A. B. Lentsch, and J. D. Richardson, "The rationale for esophagectomy as the optimal therapy for Barrett's esophagus with high-grade dysplasia," Annals of Surgery, vol. 223, no. 5, pp. 585-591, 1996.

[8] M. F. Reed, G. Tolis Jr., B. H. Edil et al., "Surgical treatment of esophageal high-grade dysplasia," Annals of Thoracic Surgery, vol. 79, no. 4, pp. 1110-1115, 2005.

[9] V. J. A. Konda, A. S. Ross, M. K. Ferguson et al., "Is the risk of concomitant invasive esophageal cancer in high-grade dysplasia in Barrett's esophagus overestimated?" Clinical Gastroenterology and Hepatology, vol. 6, no. 2, pp. 159-164, 2008.

[10] T. W. Rice, G. Zuccaro Jr., D. J. Adelstein, L. A. Rybicki, E. H. Blackstone, and J. R. Goldblum, "Esophageal carcinoma; depth of tumor invasion is predictive of regional lymph node status," Annals of Thoracic Surgery, vol. 65, no. 3, pp. 787-792, 1998.

[11] N. J. Shaheen, P. Sharma, B. F. Overholt et al., "Radiofrequency ablation in Barrett's esophagus with dysplasia," New England Journal of Medicine, vol. 360, no. 22, pp. 2277-2288, 2009.

[12] J. R. Rees, P. Lao-Sirieix, A. Wong, and R. C. Fitzgerald, "Treatment for Barrett's oesophagus," Cochrane Database of Systematic Reviews, no. 1, Article ID CD004060, 2010.

[13] C. Ell, A. May, O. Pech et al., "Curative endoscopic resection of early esophageal adenocarcinomas (Barrett's cancer)," Gastrointestinal Endoscopy, vol. 65, no. 1, pp. 3-10, 2007.

[14] E. S. Orman, N. Li, and N. J. Shaheen, "Efficacy and durability of radiofrequency ablation for barrett's esophagus: systematic review and meta-analysis," Clinical Gastroenterology and Hepatology, vol. 11, no. 10, pp. 1245-1255, 2013.

[15] J. A. Evans, D. S. Early, V. Chandraskhara et al., "The role of endoscopy in the assessment and treatment of esophageal cancer," Gastrointestinal Endoscopy, vol. 77, no. 3, pp. 328-334, 2013.

[16] R. C. Fitzgerald, M. di Pietro, K. Ragunath et al., "British Society of Gastroenterology guidelines on the diagnosis and management of Barrett's oesophagus," Gut, vol. 63, no. 1, pp. 742, 2014.

[17] B. J. Dunkin, J. Martinez, P. A. Bejarano et al., "Thin-layer ablation of human esophageal epithelium using a bipolar 
radiofrequency balloon device," Surgical Endoscopy, vol. 20, no. 1, pp. 125-130, 2006.

[18] C. Jayasekera, A. C. Taylor, P. V. Desmond, F. Macrae, and R. Williams, "Added value of narrow band imaging and confocal laser endomicroscopy in detecting Barrett's esophagus neoplasia," Endoscopy, vol. 44, no. 12, pp. 1089-1095, 2012.

[19] M. A. Kara, M. Ennahachi, P. Fockens, F. J. W. ten Kate, and J. J. G. H. M. Bergman, "Detection and classification of the mucosal and vascular patterns (mucosal morphology) in Barrett's esophagus by using narrow band imaging," Gastrointestinal Endoscopy, vol. 64, no. 2, pp. 155-166, 2006.

[20] P. Sharma, R. H. Hawes, A. Bansal et al., "Standard endoscopy with random biopsies versus narrow band imaging targeted biopsies in Barrett's oesophagus: a prospective, international, randomised controlled trial," Gut, vol. 62, no. 1, pp. 15-21, 2013.

[21] M. Singh, N. Gupta, S. Gaddam et al., "Practice patterns among U.S. gastroenterologists regarding endoscopic management of Barrett's esophagus," Gastrointestinal Endoscopy, vol. 78, no. 5, pp. 689-695, 2013.

[22] J. A. Abrams, R. C. Kapel, G. M. Lindberg et al., "Adherence to biopsy guidelines for Barrett's esophagus surveillance in the community setting in the United States," Clinical Gastroenterology and Hepatology, vol. 7, no. 7, pp. 736-742, 2009.

[23] W. L. Curvers, F. P. Peters, B. Elzer et al., "Quality of Barrett's surveillance in The Netherlands: a standardized review of endoscopy and pathology reports," European Journal of Gastroenterology and Hepatology, vol. 20, no. 7, pp. 601-607, 2008.

[24] C. Bennett, N. Vakil, J. Bergman et al., "Consensus statements for management of Barrett's dysplasia and early-stage esophageal adenocarcinoma, based on a Delphi process," Gastroenterology, vol. 143, pp. 336-346, 2012.

[25] G. R. Cameron, C. S. Jayasekera, R. Williams, F. A. Macrae, P. V. Desmond, and A. C. Taylor, "Detection and staging of esophageal cancers within Barrett's esophagus is improved by assessment in specialized Barrett's units," Gastrointestinal Endoscopy, vol. 80, no. 6, pp. 971.e1-983.e1, 2014. 


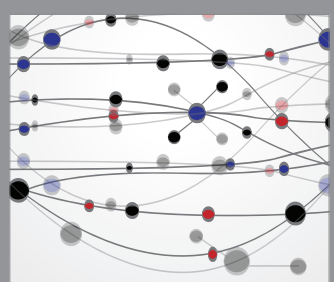

The Scientific World Journal
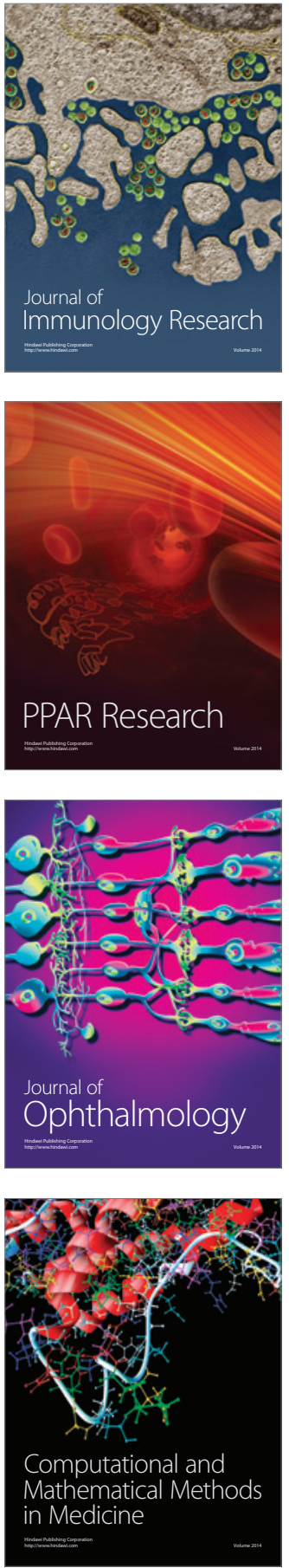

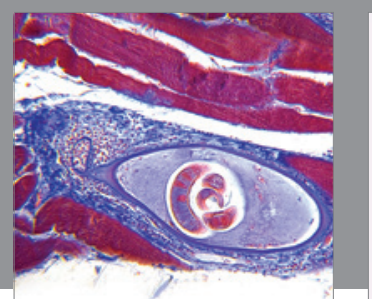

Gastroenterology Research and Practice

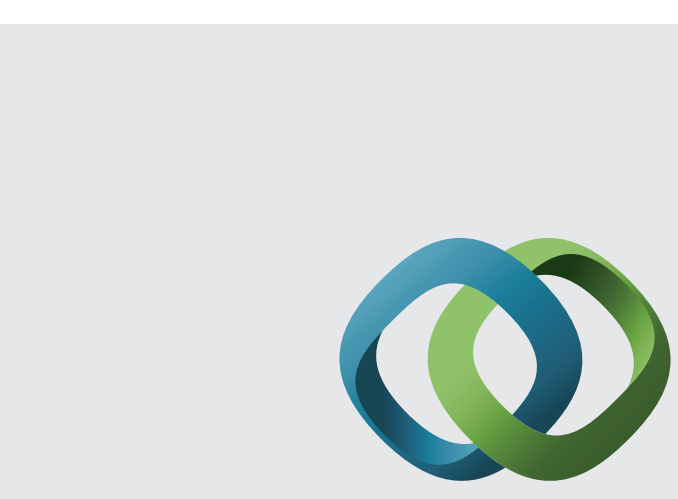

\section{Hindawi}

Submit your manuscripts at

http://www.hindawi.com
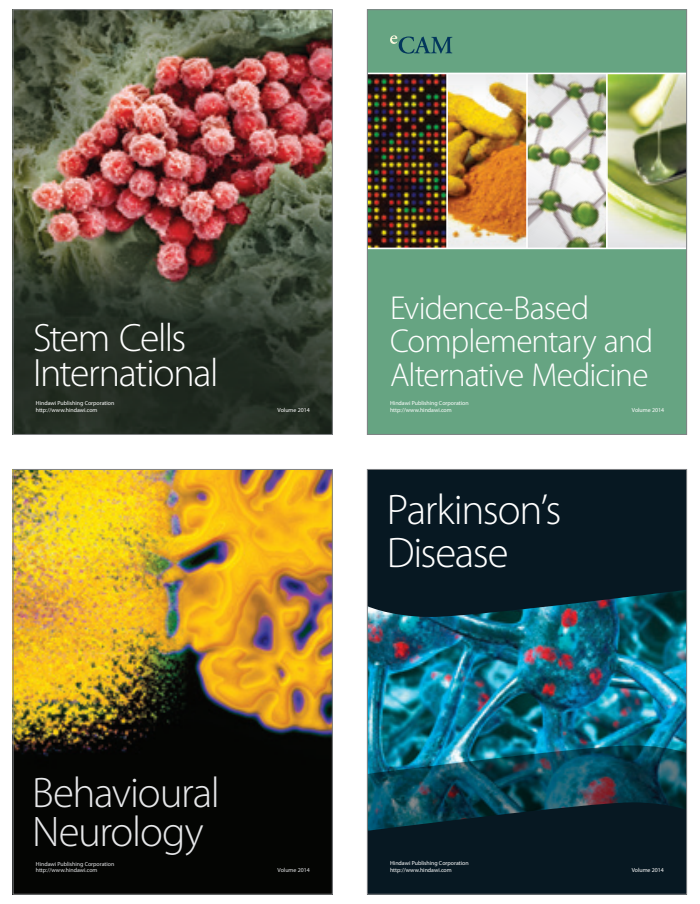
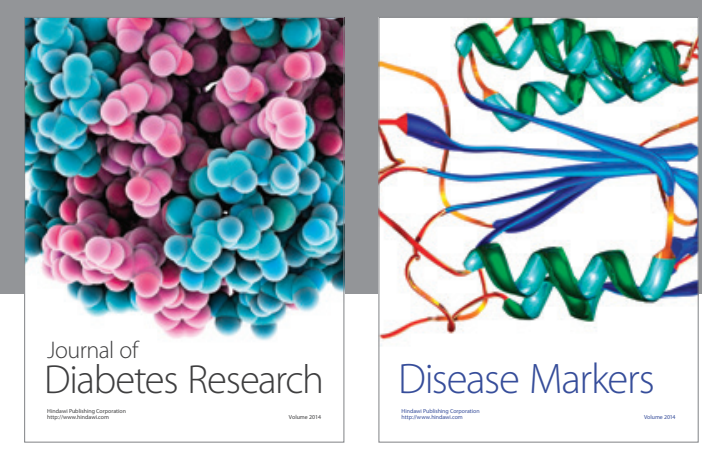

Disease Markers
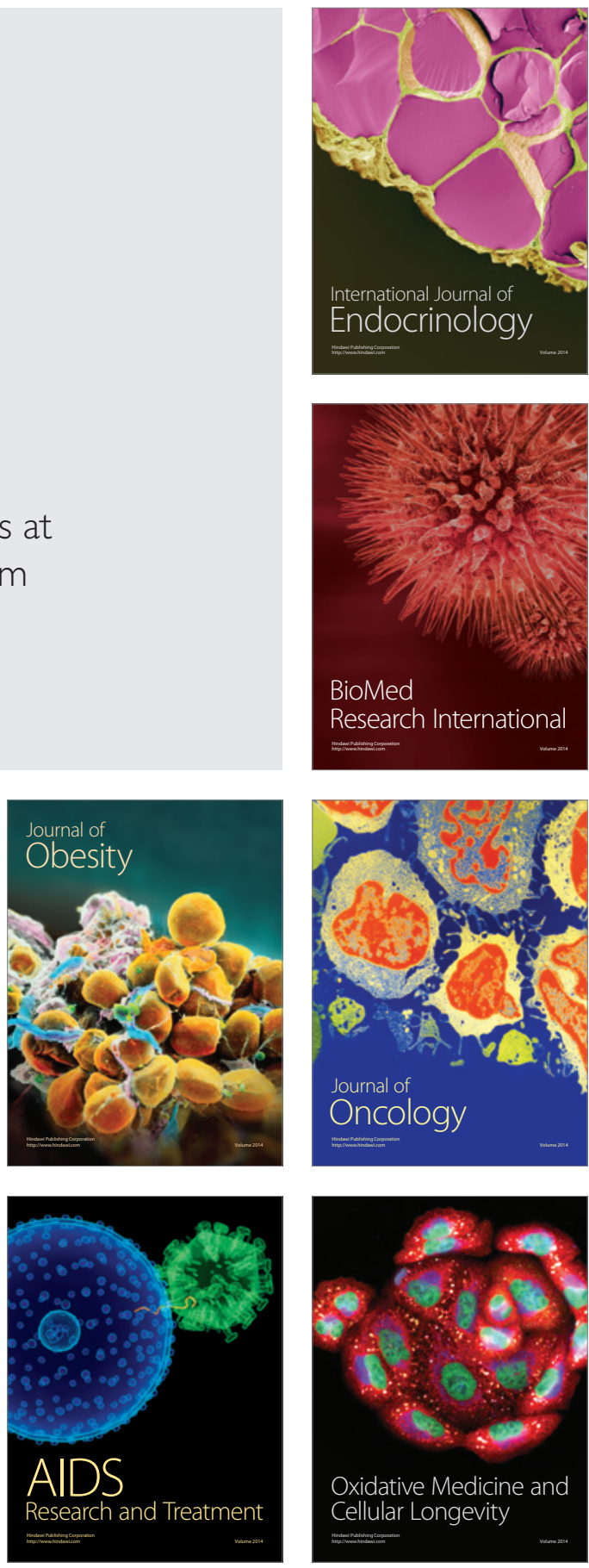